(i.e autoanitbody profile and internal organ involvement) and its impact on survival. METHODS/STUDY POPULATION: We measured serum E2 levels in $83 \mathrm{dcSSc}$ men $>50$ years old from the University of Pittsburgh Scleroderma Center and healthy controls of similar age. Using statistical modeling, we examined the associations between circulating E2 levels, internal organ involvement, autoantibody profiles, and survival. RESULTS/ANTICIPATED RESULTS: Male dcSSc patients had significantly higher serum E2 levels compared to healthy male controls and compared to dcSSc post-menopausal women of similar age. Male dcSSc patients with high serum E2 levels had significantly more heart involvement and worse survival. Using Cox regression modeling for risk of death, increasing serum E2 levels in anti-Scl-70 antibody positive dcSSc males were associated an increased risk of death. DISCUSSION/ SIGNIFICANCE OF IMPACT: DcSSc male patients have higher levels of E2 compared to healthy controls and dcSSc postmenopausal women. Elevated serum E2 levels in dcSSc males $>50$ are associated with heart involvement and, if anti-Scl-70 antibody positive, worse survival. Our current study expands on our previous work, not only that that E2 exerts pro-fibrotic effects on skin, but also internal organ involvement, overall survival. These data suggest an important role of estrogen imbalance in SSc.

3248

\author{
Fluorescence-Guided Neurosurgery with \\ 5-Aminolevulinic-Acid and \\ Second-Window-Indocyanine-Green: A murine model \\ and investigation into suitable cell lines. \\ Steve Cho ${ }^{1}$, John Y.K. Lee and Saad Sheikh \\ ${ }^{1}$ University of Pennsylvania School of Medicine
}

OBJECTIVES/SPECIFIC AIMS: This study aims to understand the utility of 5-ALA and SWIG in detecting areas of neoplasm in a murine model of GBM. Primary outcome is the distribution of the two dyes in comparison to the true tumor extent; the sensitivity, specificity, PPV, and NPV of both dyes will be calculated. The secondary outcome is the suitability of existing cell-lines used for GBM research for studies in fluorescence-guided surgery. METHODS/ STUDY POPULATION: Two cell lines are used for this research: U87, derived from human GBM; and GL261, derived from rodent stem cells. U87 are implanted intracranially into 6-week old athymic, nude, female mice, while GL261 are implanted intracranially into 10 -week old female C57BL/6 mice. The mice are weighed every 3 days to monitor health and bioluminescence imaging is performed between 7-10 days after implantation to confirm tumor implantation and monitor tumor growth. The mice are sacrificed between 10-21 days after implantation. $5 \mathrm{mg} / \mathrm{kg}$ of intravenous ICG is administered 24 -hours prior to harvest and $250 \mathrm{mg} / \mathrm{kg}$ of intraperitoneal 5-ALA is administered 3-hours prior to harvest. Once the mice are sacrificed, their brains are quickly harvested and placed in cold formalin. Using a high-resolution Odyssey CLx scanner, near-infrared fluorescence from ICG is captured in coronal cross sections of the brains through the tumor. Similarly, 5-ALA fluorescence is imaged using a $405 \mathrm{~nm}$ LED excitation source and 610-690nm bandpass filter. Afterwards, slices of the brain are stained with H\&E, which serves as the goldstandard of the extent of tumor. Images from ICG, 5-ALA, and $\mathrm{H} \& \mathrm{E}$ can then be compared using ImageJ to compare the extent of tumor to the distribution of the dyes. RESULTS/ANTICIPATED RESULTS: In separate, previous studies in humans, both 5-ALA and SWIG have demonstrated utility in detecting residual neoplasm in HGG resections. In general, 5-ALA is more specific for areas of neoplasm, while SWIG is more sensitive. Thus, I anticipate that in this study, SWIG will show a greater distribution than 5-ALA, with SWIG distributing to areas beyond the tumor and 5-ALA distributing within, but not completely covering, the tumor. SWIG's sensitivity and NPV for detecting tumor should be $>90 \%$, while its specificity and PPV may be closer to 50\%. For 5-ALA, specificity and PPV should be close to $80-90 \%$, but its sensitivity and NPV may be $<50 \%$. In terms of cell-line, preliminary results suggest that U87 cells are not suitable for research involving 5-ALA. We suspect that this is partly due to the limited infiltrative nature of U87 cells; in fact, the cells form a spherical mass, imitating metastases rather than true HGGs. The U87 masses do not have significant vascularity, which likely limits the amount of 5-ALA that can distribute to inside the tumor. DISCUSSION/SIGNIFICANCE OF IMPACT: 5-ALA is currently the only FDA-approved agent for fluorescence-guided neurosurgery. However, it has multiple limitations, which ultimately results in its low sensitivity and NPV. Our novel technique, which has demonstrated much higher sensitivity at the cost of specificity, offers an alternative that may help surgeons better achieve total resections in the operating room. These two agents have not been compared directly in humans or mice. Thus, this experiment sets up an important precedent, on which a human clinical trial comparing the two agents' effects on resection rates and patient outcome can be performed. Ultimately, this work will lay the foundation for future research into fluorescence-guided neurosurgery, both in the visible and NIR spectrum.

Fracture Targeted Parathyroid Hormone Agonist As An Effective Pharmaceutical For Bone Repair in Mouse and Canine Models

Jeffery Jay Howard Nielsen ${ }^{1}$, Stewart A. Low and Philip S. Low

${ }^{1}$ Purdue University; Purdue University

OBJECTIVES/SPECIFIC AIMS: The primary objective of this study was to evaluate the performance of a bone fracture targeted systemically administrable bone anabolic as a potential therapeutic for bone fracture repair. Currently all bone fracture repair therapeutic require local administration during surgery. However, the population that need the most assistance in repair bone fractures are not eligible for surgery. So, it was our goal to design an inject-able therapeutic to assist in bone fracture repair to reduce the invasiveness. The injectable nature of it allows for repair administration of the bone anabolic and for therapeutic effect throughout the entire bone fracture healing process. Targeting it to the bone fracture site reduces the toxicity and increases the efficacy. METHODS/STUDY POPULATION: METHODS To achieve the above objective, a bone mineral-(hydroxyapatite-) targeting oligopeptide was conjugated to the non-signaling end of an engineered parathyroid hormone related protein fragment 1-46 with substitutions at Glu22,25, Leu23,28,31, Aib29, Lys26,30 (ePTHrP). The negatively charged oligopeptide has been shown to target raw hydroxyapatite with remarkable specificity, while the attached PTHrP has been demonstrated to induce sustained and accelerated bone growth under control of endogenous morphogenic regulatory factors. The conjugate's specificity arises from the fact that raw hydroxyapatite is only exposed whenever a bone is fractured, surgically cut, grafted, or induced to undergo accelerated remodeling. The hydroxyapatite-targeted conjugate can therefore be administered systemically (i.e. without invasive surgery or localized injection) and still accumulate on the exposed hydroxyapatite at the fracture site where it accelerates the healing process 
Murine in vivo experiments were conducted on female Swiss Webster mice (10 per group). Femoral fractures were induced with a 3-point bending device and stabilized. Mice were dosed with $3 \mathrm{nmol} / \mathrm{kg} / \mathrm{d}$ of targeted-ePTHrP, non-conjugated (free) ePTHrP, or saline. Following a 4-week study, fracture callus densities were measured using microCT. Canine in vivo experiments were conducted on 1-year-old male beagles. Beagles underwent a $10 \mathrm{~mm}$ bilateral ulnar ostectomy. Two dogs in the treatment group and Three dogs in the control group were dosed daily with either targetedePTHrP $0.5 \mathrm{nmol} / \mathrm{kg} / \mathrm{d}$ or saline respectively. Dogs were $\mathrm{x}$-rayed weekly for the first 6 weeks and then every other week thereafter. One tailed ANOVA followed by Dunnett's post-hoc test was used to establish significance. All animal experiments were conducted as described in approved IACUC protocols. $\mathrm{P}<0.05$ was considered significant. RESULTS/ANTICIPATED RESULTS: RESULTS SECTION: In the murine studies we observed a marked increase in fracture callus size and a 2 -fold increase in bone deposition was observed in the targeted-ePTHrP group over the saline group $(\mathrm{P}<0.01)$. A significant doubling in bone density was also observed. Targeted-ePTHrP group fractured femurs were able to achieve their pre-fracture strength as early as 3 weeks compared to 9 weeks in the saline mice representing a $66 \%$ reduction in healing time. In the canine studies, we observe a significantly higher closure of the ostectomy gap than saline controls $(\mathrm{P}<0.05)$. In addition, no significant differences in weight are observed in the treatment vs. saline controls. No significant difference between the control group and treatment groups was found in a histological investigation of the organs. DISCUSSION/SIGNIFICANCE OF IMPACT: DISCUSSION: Although attempts have been made in developing a systemically administered fracture therapeutic for fracture repair, i.e. teriparatide, to date, no such anabolics have been approved for this use. In these studies there is evidence that anabolic activity was occurring at the fracture site, but at a level that did not meet FDA required end-points. 2 It is plausible that if sufficient drug were to be delivered to a fracture site then improved fracture repair would be possible. In previous studies, we demonstrated fracture specific accumulation bone anabolics can be achieved by modifying the drug with acidic oligopeptides. 3 Here, by modifying a safe, clinically proven, parathyroid hormone receptor agonist with an acidic oligopeptide we observe improved bone deposition and strength in mice. Furthermore, when administered to canine critical sized defect ostectomies, a more relevant and difficult model, we observe improved ostectomy closure. CLINICAL RELEVANCE:: The ability to accelerate bone fracture repair is a fundamental need that has not been addressed by conventional methods. By targeting bone anabolic agents to bone fractures, we can deliver sufficient concentrations of anabolic agent to the fracture site to accelerate healing, thus avoiding surgery and any ectopic bone growth associated with locallyapplied bone anabolic agents.

3463

\section{Frontal Alpha Asymmetry in Alcohol-Related Intimate Partner Violence}

Brandi C Fink, PhD

University of New Mexico Clinical and Translational Science Center

OBJECTIVES/SPECIFIC AIMS: The current study is the first investigation of frontal alpha asymmetry in distressed violent (DV) and distressed nonviolent (DNV) partners during a placebo-controlled alcohol administration and emotion-regulation study. Because this is the first study of the pharmacological effects of alcohol on FAA, the first portion of the study was conducted to characterize alcohol effects in DV and DNV partners during the baseline condition. The subsequent portions of the study were conducted to characterize the effects of alcohol and evocative stimuli on FAA in DV and DNV partners. We hypothesized that DV partners would demonstrated greater left frontal alpha asymmetry when intoxicated and viewing evocative partner stimuli than DNV partners. Lastly, we attempted to replicate previous research that has found associations between baseline measures of FAA and the State-Trait Anger Expression Inventory - 2 (Spielberger, 1999) subscales of Trait Anger, Anger Expression-Out, Anger Expression-In, Anger ControlOut, Anger Control-In (Hewig, Hagemann, Seifert, Naumann, \& Bartussek, 2004). METHODS/STUDY POPULATION: Partners in the present study were drawn from a larger study investigating over-arousal as a mechanism between alcohol use and intimate partner violence (AA022367). Couples were recruited from the community via radio, television and newspaper advertisements, and eligibility screening occurred at the couple level. Participants included in the present analysis were $23 \mathrm{DV}$ partners ( 12 female, 11 male), and $15 \mathrm{DNV}$ partners (7 female, 9 male). The mean age of the sample was 32 (SD 4.8 years, range $23-40$ years). Data from two DV partners were not included in the analyses of the FAA in the emotion-regulation tasks due to movement artifacts during the alcohol condition leaving too little data for analysis. RESULTS/ANTICIPATED RESULTS: The expected beverage by couple type interaction did not reach significance $[\mathrm{F}(1$, $36)=3.93, p=.055]$, but the between-subjects effects of couple type revealed a significant difference $[\mathrm{F}(1,36)=4.425, \mathrm{p}=.042]$. Contrary to our hypothesis, however, these results suggest that under conditions of alcohol, DV partners evidenced significantly greater relative right frontal alpha power asymmetry whereas DNV partners evidenced greater relative left frontal alpha power asymmetry. Although there was no significant between-subjects effect, there was a nearly significant interaction between beverage type and emotion regulation condition $[F=(1,36)=4.032$, $\mathrm{p}=.052]$ and a significant main effect of emotion regulation condition $[\mathrm{F}(1,36)=7.579, \mathrm{p}=.009]$. It appears that asking the participants to "not react" to their partners' evocative stimuli caused significantly greater right frontal alpha asymmetry. Because intimate partner violence is best understood in the context of conflict between two partners, we also examined partner-reported experiences of anger as predictors of DV participant's FAA. The model as a whole predicted $67.4 \%$ of the variance in $\mathrm{DV}$ partner FAA, R squared change $=.674$, F Change $(5,15)=6.21, \mathrm{p}=.003$. Three anger experience scales were statistically significant. The partner Anger Control-Out $(\mathrm{B}=-1.23, \mathrm{p}=.001)$ scale recorded a higher standardized beta value and accounted for $40 \%$ of the variance in this model. Anger Control-In $(B=.63, p=.022)$ accounted for $14 \%$ of the variance in the model, and Anger Expression-Out scale $(\mathrm{B}=.57, \mathrm{p}=.024)$ accounted for $13.7 \%$ of the variance in the model. DISCUSSION/ SIGNIFICANCE OF IMPACT: The current study is the first pharmacological study of the effects of alcohol on frontal alpha asymmetry in distressed violent and distressed nonviolent partners. Contrary to our hypothesis, under acute alcohol intoxication during the baseline condition, DV partners exhibited significantly greater relative right FAA compared to DNV partners who exhibited significantly greater relative left FAA. Because intimate partner violence is best understood in the context of couple conflict, we examined the ability of partners' anger experiences to predict DV and DNV partners' FAA, and a very interesting pattern emerged among our DV participants and their partners. The anger experiences of our DV participants' partners accounted for $67 \%$ of the variance in the FAA of our DV participants when they were intoxicated and viewing evocative stimuli. 